Gynecol Obstet Invest 1997;44:281-282

\title{
Author Index Vol. 44, 1997
}

Gynecologic and Obstetric Investigation

Abramovici, H. 53 Abu-Heija, A.T. 169 Acevedo,C.G. 153 Albrecht,A. 127 Alevizaki, C.C. 6 Alevizaki, M. 6 Alge,A. 206 Altaras, M.M. 200 Anastasiou, E. 6 Andersch,B. 16,224 Angeletti, G. 141 Arduini, D. 249 Arslan, A. 112 Asada,Y. 47

Baiocchi, G. 141 Baldwin, S. 260 Baranowski, W. 132 Batioglu, S. 64 Beek,J.F. 115 Berg, R.W. 260 Bergholz,C. 260 Bernheim, J. 200 Bertat,R. 191 Beyth, Y. 200 Bianciotto, A. 107

Biberoglu,K. 1 Blanck,A. 93,221 Blickstein, I. 244 Bornstein, J. 53 Bots, R.S.G.M. 149 Bracero, L.A. 21 Bravo, I. 153 Brockman, D.E. 177

Calabrese, O. 107 Calderon, I.M.P. 11 Cappellini, A. 278 Celleno,R. 141 Chang, A.M.Z. 136,229 Chatenoud,L. 239 Cheng, X. 57 Chervenak, FA. 244 Cheung, T.H. 136 Chung, T.K.H. 187 Ciavattini, A. 234,249 Cipriano, L. 67 Cline,J.M. 41 Cohen, I. 200 Colamaria, A. 214 Contreras, E. 153 Çorakçi, A. 112

Cordoba, M. 200 Cormio, G. 214,278 Covello,R. 67 Crosignani, P.G. 239

Dani§man, N. 1 De Cristofaro, F. 234, 249 De Lauretis, L. 239 DiVagno,G. 214 Dollberg, S. 177 Duley,J $\Lambda .229$

Ehn,B.E. 182 Ekici,E. 64 El-Jallad, M.F. 169 El-Sunna,E. 169 Enane, N.A. 260 Endoh,M. 275 Erhan,G. 112 Evers,J.L.H. 149

Farage-Elawar, M.A. 260 Farina, A. 107 Faticanti Scucchi, L. 67 Faxén,M. 93,221 Fishman, A. 200 Foth,D. 124 Fratini,D. 141 Frega, A. 67 Frigo,P. 211 Furuya,K. 102

Garzetti, G.G. 234, 249 Gemert, M.J.C. van 115 Güardi,G. 141 Giorgetti, M. 107 Goldman, R.D. 244 Grabenwöger, F. 211 Grigorakis, S.I. 6 Gschwendtner, A. 206 Gurewitsch, E. 244 Haberal, A. 64 Hachisuga, T. 32 Haines, C.J. 187 Halligan, A.W.F. 38 Hara,M. 275 Hartmann, B.W. 127 Hashimoto, S. 275 Hata,K. 173 Hata,T. 173 Hattori, S.-e. 270

Hayata,T. 70 Heinonen, P.K. 89 Hidaka,A. 196 Hirota,Y. 70 Hoist, E. 16 Hseuh,C. 61 Hsieh,C.C. 61 Huambachano, A.M. 153 Huber,J.C. 127

Ikeda,M. 275 Ino,Y. 47 Inoue, T. 270 Irie, T. 57 Ishikawa,H. 270 I§ik,Z. 1 Itamochi, H. 57 Ito,M. 82 Iwasaka, T. 32 Izumi, A. 26

Jakowicki, J.A. 132 Järvinen, T. 89 Jorizzo, G. 107 Joura, E. 211

Kaaijk, E.M. 115 Kahraman, S. 1 Kainulainen, H. 89 Kalahanis,J. 120 Kanamori, Y. 57 Kaplan, B. 255 Kato,T. 102 Kawabata, M. 196 Kawano, Y. 145 Kigawa, J. 57 Kikkawa,F. 270

Kirchengast, S. 127 Koike, T. 97 Kölbl,H. 211 Kreczy, A. 206 Kudoh,K. 102 Kuwata,T. 97 Kuwayama, T. 47

La Marca, N. 234, 249 Laml,T. 127 Lammes, F.B. 115 Lang, C. 211 Lau,T.K 187 Lee,T. 196 Liang, C.-C. 61

\section{KAílGER}

(C) 1997 S. Karger AG, Basel

281

E-Mail karger@karger.ch Fax + 41613061234 http://www. karger. ch 
Liljestrand, J. 182 Lindstedt, G. 224 Lundberg, P.-A. 224 Lunell,N.-O. 93,221 Lurie, S. 255 McParland, P. 38 Maeda,Y. 82 Mairinger, T. 206 Makihara,K. 173 Makimura, N. 102 Manabe, A. 173 Manci, N. 141 Masaoka, N. 82 Matsuda, Y. 82 Matsui,N. 145 Matsumoto, Y. 32 Matsuzawa, K. 270 Mavromatidis, G. 120 Mercan, R. 112 Milsom,I. 16,224 Minagawa, Y. 57 Minaguchi, H. 275 Minakami, H. 26, 97 Miturski, R. 132 Miyakawa, I. 70, 145 Miyamura, K. 70, 145 Miyazaki, K. 173 Mizuno, K. 47 Mizutani, S. 47 Mollica,G. 107 Mongelli,M. 229 Mori,E. 157 Morioka,Y. 157 Myatt,L. 177

Nadaoka,T. 157 Naftalin, N.J. 38 Nagata, I. 102 Nakamura, K. 32 Narahara, H. 145 Nasiell,J. 93 Nasu,K. 145 Neri,A. 255 Nilsson,C. 16 Nomiyama, M. 32 Nuhoglu, A. 1

Obeidat,A. 169 Ogawa, S. 97 Okada,M. 47,57

Özeren, S. 112 Özgün, O.D. 1

Palm,C. 163 Pang,C.P. 229 Panidis,D. 120 Parazzini, F. 239 Peeters,M.F. 149 Peled,Y. 255

Pellegrino, A. 278 Peraçoli, J.C. 11 Perego, P. 278 Perotti,L. 239 Petropoulos, P. 120 Petters, R. 191 Pierangeli, E. 214 Pim,A. 11 Poppers, P.J. 73

Rabinerson, D. 255 Ragni,G. 239 Ramos, M.D. 11 Rapti,E. 6 Reis,F.M. 191 Rogers, M.S. 136,229 Römer,Th. 124 Rossel Goffeng, A. 16,224 Rossi, R. 278 Rousso, D. 120 Rousso, I. 120 Royburt,M. 255 Rudge,M.V.C. 11 Rydhstroem, H. 163

Saito,H. 157 Sakamoto, H. 82 Sapienza, F. 239 Sarbaugh, F.C. 260 Sato, I. 26, 97 Sato,K. 82 Schabel,J.E. 73 Schoultz, B. von 41 Seibel, M.M. 217 Seki,K. 102 Sekiya, S. 102 Selvaggi,L. 214 Semczuk, A. 132 Shafik,A. 265 Shapira,J. 200 Shatnawi,A. 169 Shirasu, K. 275

Skiadopoulos, S. 120 Skoog,L. 41 Smith-Levitin, M. 244 Söderqvist, G. 41 Soelder, E. 206 Soong,Y.-K. 61 Söregi, G. 127 Sou,H. 32 Souvatzoglou, A. 6 Stentella, P. 67 Streltzhoff, J. 244 Suganuma, N. 270 Sugimori, H. 32 Suzuki, A. 47

Taga,M. 275 Takada,M. 82 Takayama, T. 97 Tamakoshi, K. 270 Taylor, D.J. 38 Tepper,R. 200 Terakawa, N. 57 Tiemessen, C.H.J. 149 Tokuhashi,Y. 270 Tomoda,Y. 47,270 Tsai,C.-C. 61 Tsuda,H. 196

Uemura, T. 275

Vailati,G. 214 Veen, F. van der 115 Vegetti,W. 239 Vesce,F. 107 Vicdan,K. 1 Vilodre, L.-C.F. 191

Wang,W. 229 Wihman,I. 221 Wong,Y.F. 136

Yamamoto, K. 196 Ye§ilyurt, H. 64 Yigael,D. 200 Yim,S.F. 136 Yücesoy, í. 112

Ziadeh,S. 169 Zilberstein, M. 217

282

Gynecol Obstet Invest Vol. 44, 1997

Author Index 